\title{
Performance Analysis of Downlink and Uplink Transmission Schemes in 4G LTE Using Various Modulation Techniques
}

\author{
Owk. Srinivasulu ${ }^{1,}$ Prof .P. Rajesh Kumar ${ }^{2}$, \\ ${ }^{1}$ Associate professor, St. Peter's Engineering College-Hyd. Research scholar, AUCE, visakapatnam-03, \\ ${ }^{2}$ Professor\& Head of the Department, Department of ECE, AUCE, visakapatnam-03
}

\begin{abstract}
In the growth of fourth-generation mobile servicing, long ` $\mathrm{c} n \mathrm{~ns}$
term evolution (LTE) is an emanate technology. In LTE the downlink model build up on orthogonal frequency division multiple access (OFDMA) and the uplink arrangement build up on single carrier frequency division multiple access (SC-FDMA).In this paper we have tendency to derive the achievement of typical elemental differences between the OFDMA and SC-FDMA schemes. In the cutting-edge wireless mobile communications multiple access schema should meet certain requirements like low bit error rate (BER), high signal-to-noise ratio(SNR), high throughput, high spectral efficiency, good channel capacity, low peak to average power ratio(PAPR) and high quality etc. In this paper the act of OFDMA and SC-FDMA in LTE physical layer are reviewed by using various modulation techniques (BPSK, QPSK and 16QAM) with respective to SNR, BER, and $P A P R$. Finally it summarizes the simulation results of OFDMA and SC-FDMA transmission modes.
\end{abstract}

Key Words: LTE, OFDMA, SC-FDMA, BER, SNR and PAPR.

\section{Introduction}

International Telegraph Union (ITU) (currently, International Telecommunication Union) has been involved to standardize the telecommunication universally from telegraph to today $4^{\text {th }}$ generation systems. As of late, in 2000, to fulfill the ITU's third era portable framework norms, an association of 3GPP was shaped. The 3rd Generation Partnership project is a coordinated effort of gatherings of telecom affiliations taking a shot at Global System for Mobile Communication (GSM).3GPP distributed and presented the different gauges for IP based framework in Release 8, which was additionally named Long Term Evolution and condensed as LTE. As of late in 2011, LTE was additionally created through Release 10 to fulfill ITU's IMT-Advanced necessities for 4G cell frameworks. LTE radio transmission and gathering determinations are recorded in TS 36.101 for the UE (User Equipment) and TS 36.104 for the eNB (Evolved Node B). According to these determinations, LTE is equipped for supporting up to 1Giga Bits for every second (1Gbps) for repaired client and to $100 \mathrm{Mbps}$ for rapid client. The prime reason for this fast of LTE frameworks is the progression in physical layer LTE was started as a scheme in 2004 by media transmission body known as the Third Generation Partnership Project (3GPP). The term LTE is ordinarily used to speak to both LTE and SAE.LTE advanced from a before 3GPP framework known as the Universal Mobile Telecommunication System UMTS, which thus developed from the Global System for Mobile Communications (GSM). Indeed, even related particulars were formally known as the developed UMTS earthly radio get to E - UTRA and advanced UMTS earthbound radio get to network E UTRAN. Initially form of LTE was recorded in Release 8 of the 3GPP determinations. A fast increment of versatile information use and development of new applications, for example, MMOG Multimedia Online Gaming, portable TV, Web 2.0, gushing substance have spurred the third Generation Partnership Project 3GPPto work on the Long-Term Evolution LTE in transit towards fourth era versatile. The principle objective of LTE is to give a high information rate, low inactivity and parcel improved radio get to innovation supporting adaptable data transmission organizations

\section{Literature Survey}

$1 \mathrm{G}$ was actually meant for voice services, the technology used in this is FDMA. The AMPS (Advance Mobile Phone System) in United States and the TACS (Total Access Communication System) in many parts of Europe comes under this 1G.Be that as it may, AMPS established the framework to the "cell" innovation which spearheaded the utilization of little hexagonal cell regions and henceforth upheld recurrence re-use over the "cells" without obstruction. The $1 \mathrm{G}$ innovation was soon supplanted by the second era or $2 \mathrm{G}$ advances which spoke to the substitution of the simple radio system with computerized radio system. $2 \mathrm{G}$ is actually meant for voice and data. 2G advancements could be additionally ordered into Time Division Multiple Access (TDMA) based and Code Division Multiple Access (CDMA) based. The TDMA based innovation was received generally in Europe and was called Global framework for Mobile correspondences or GSM (Global system for mobile communications) while USA embraced the CDMA based innovation and it was called CDMA one or institutionalized as Seems to be 95a. CDMA had the upside of supporting a bigger number of clients than GSM 
because of the better utilization of the range. CDMA is a spread range innovation in which every client is permitted to transmit over the entire range utilizing an alternate orthogonal code.. CDMA one bolstered computerized information exchange rates ranging 4.8-14.4 kbps while CDMA two upheld signaling bits approximately $115.2 \mathrm{kbps}$. The2nd generation innovation prompted a between time era of $2.5 \mathrm{G}$ which spoke to $2 \mathrm{G}$ frameworks which executed a parcel exchanged Neighboring cells may reuse a similar recurrence band and not meddle the length of the utilize diverse code, in this area notwithstanding the circuit exchanged space. General Packet Radio Service (GPRS) was the $2.5 \mathrm{G}$ innovation embraced by global system by mobile communications. General pocket radio system gives parcel exchanged administration over GSM expiation information rates 56-114 kilobits per sec.

Improved data rates for GSM evolution (EDGE) over GSM and CDMA2000 1xRTT over CDMA was considered as $2.75 \mathrm{G}$ innovations however they may be called $3 \mathrm{G}$ advances as the outperform information speeds of 144 kilobits per sec. Enhanced data rates for GSM evolution gives information speeds of 236.8 kilobits per sec while code division multiple access 2000 technology constrain the information rates at $144 \mathrm{kbps}$. The International Telecommunication Union (ITU) under the International Mobile Telecommunications Program settled the base information rate of $144 \mathrm{kbps}$ for any innovation to fit the bill to be a $3 \mathrm{G}$ innovation. Notwithstanding, most advances which fall under this class by a wide margin outperform this base breaking point and give information rates normally between 5-10 Mbps. 3G Technologies achieve better ghostly effectiveness (more bits/Hertz) over wide region cell phone systems taking into consideration higher information rates and improved administrations. The primary pre-business and business $3 \mathrm{G}$ innovation was introduced in Japan took after by South Korea. In Europe the Universal Mobile Telecommunication System (UMTS) is the embraced 3G innovation utilizing W-CDMA (Wideband Code Division Multiple Access) as the air interface. UMTS is here and there called 3GSM to underline the way that it is the third era innovation succeeding GSM. The advancement of CDMA based advances to 3G was through the CDMA2000 group of conventions, particularly EV-DO (Evolution-Data Optimized) which utilizes multiplexing methods including CDMA and TDMA to increment per client and additionally framework throughput. UMTS based 3G innovations have raised themselves to 3.5G with HSDPA (High Speed Downlink Packet Access) permitting information rates up to $7.2 \mathrm{Mbps}$. Likewise these systems are arranging their advance into $4 \mathrm{G}$ through the $3 \mathrm{GPP}$ (third Generation Partnership Projects) where they intend to accomplish information rates in the request of 100Mbps downlink and 50 Mbps Uplink.

Utilization of MIMO innovation has been presented progressively over the diverse arrivals of the LTE norms. MIMO has been a foundation of the LTE standard, yet at first, in discharges 8 and 9 various transmit reception apparatuses on the UE was not upheld on the grounds that in the intrigued of energy diminishment, just a solitary RF control enhancer was thought to be accessible. It was in Rel. 10 that various new plans were presented. Shut circle spatial multiplexing for SU-MIMO and numerous reception apparatuses on the UE. There are a few courses in which MIMO is executed in LTE. These fluctuate as per the gear utilized, the channel work and the hardware required in the connection.

\section{Design Methodology}

For uplink scheme an emerging technology of single carrier frequency division multiple access (SCFDMA) is used for high data rates and has been hugged by 3GPP for it cutting edge cell framework, called Long-Term Evolution (LTE). SC-FDMA is mainly depends on the multicarrier nature of OFDM for high throughput. To transform time domain samples to frequency domain samples this is regularly seen as DFTcoded OFDM before experiencing the standard OFDM tweak, so it receives the advantages OFDM over TDMA and CDMA. The significant issue in GSM TDMA and wideband CDMA is increasing complexity in multipath signal reception. The fundamental favorable position of OFDM, as is for SC-FDMA, is its vigor against multipath flag spread, which makes it appropriate for broadband systems. SC-FDMA conveys extra advantage of low crest to-normal power proportion (PAPR) contrasted with OFDM making it appropriate for uplink transmission by client terminals. SC-FDMA constructed on OFDM modulation technique .The block diagram of SC-FDMA is shown in below figure 1.

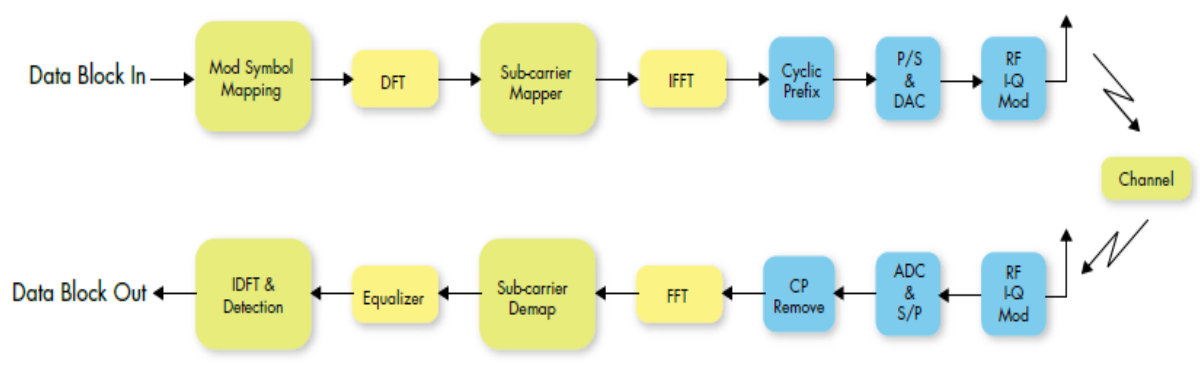

Fig 1: SC-FDMA Transmitter and Receiver 
OFDM transmitter and receiver structure is appeared in Figure. A transmitter incorporates a baseband modulator, subcarrier mapping, backwards Fourier change, cyclic prefix expansion, parallel-serial transformation, and a computerized to-simple converter taken after by an I-Q RF modulator. Dissimilar to other adjustment methods that work image by image, OFDM transmits a square of information images all the while more than one OFDM image. An OFDM image is the time used to transmit all of subcarriers that are tweaked by the piece of info information images. The baseband modulator changes the information twofold bits into an arrangement of multi-level complex numbers that relates to various adjustments organizations, for example, BPSK, QPSK, 16 or 64-QAM.

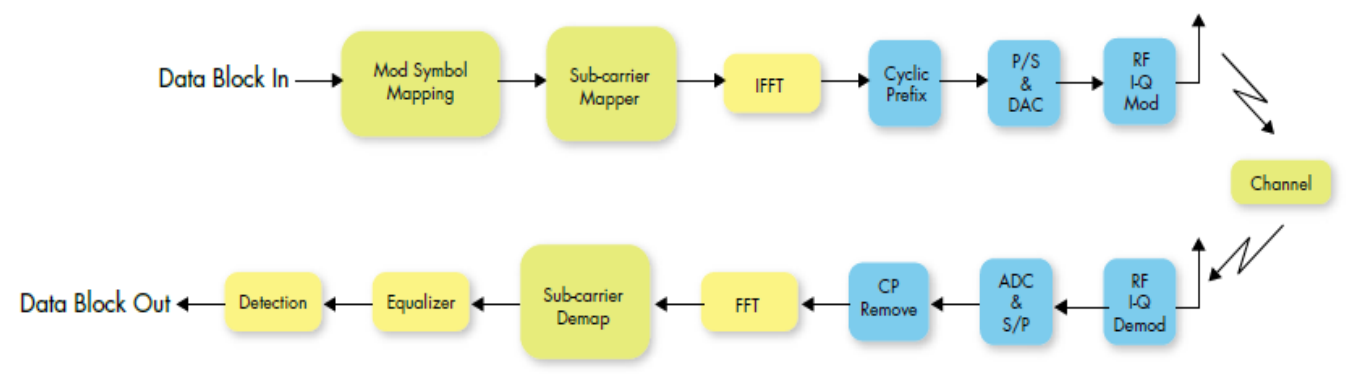

Fig 2: OFDMA Transmitter and Receiver

Based on the received signal-to noise ratio of received signal and ability of the receiver for correct decoding, kind of modulation is used. These modulated symbols are then mapped to subcarriers. Then these sub carriers are converted from frequency domain to time domain by using an inverse-FFT (IFFT). Same modulation can be used to keep the all subcarriers control information small. And also it is possible to use the different modulation formats and it is advantageous in typical time varying conditions. Over large system bandwidth the channel is frequency selective. In a broadband system, meaning each subcarrier of subcarrier signal is independent. Each sub carrier interference level is different on each sub carrier and varies uniquely with time. On each sub carrier signal to impairment level is different. So suitable modulation format on these sub carrier increases throughput of the system on the whole depending on the channel conditions adaptation of modulation schemes receives by OFDM this is called Channel-dependent scheduling. A portion of the samples copies by cyclic prefix block at the end of the time domain samples block (at the IFFT output) to the beginning. So outputs of the DFT/FFT in theory they are periodic, the samples copying to the beginning will make the signal continuous. Cyclic prefix length depends on the delay spread of the channel, and is preferably longer than the length of the channel response. At the receiver, the prefix part of the symbol is thrown away as it may contain ISI from its previous symbol. Hence, it removes the effect of ISI caused by the multipath signal propagation. However, the prefix is the overhead in an OFDM system, as it does not carry any useful information. The block of complex samples are then serialized in the time domain and converted to analog signals. The RF section modulates the I-Q samples to final transmission radio frequency. A corresponding receiver does the inverse operations of the transmitter in the reverse order. A typical OFDM receiver includes an RF section, ADC, parallel-to-serial converter, cyclic prefix remover, Fourier transformer, sub-carrier de-mapper, equalizer and detector. The main difference between OFDM and SC-FDMA transmitter is the DFT mapper. After mapping data bits into modulation symbols, the transmitter groups the modulation symbols into a block of $\mathrm{N}$ symbols. An N-point DFT transforms these symbols in time domain into frequency domain. The frequency domain samples are then mapped to a subset of $\mathrm{M}$ subcarriers where $\mathrm{M}$ is typically greater than N. Similar to OFDM, an M-point IFFT is used to generate the time-domain.

\section{Simulation Results}

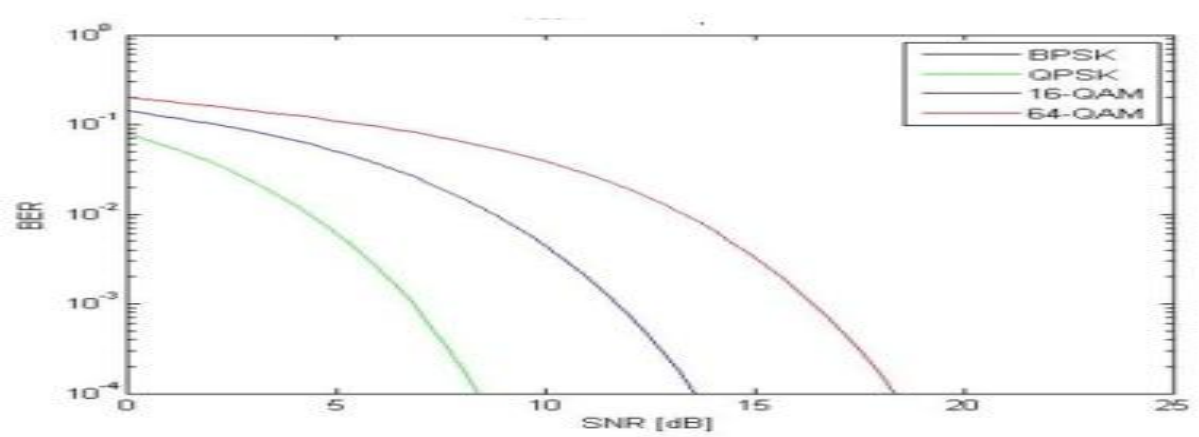

Fig 3: BER vs. SNR of OFDMA with BPSK, QPSK, 16QAM 


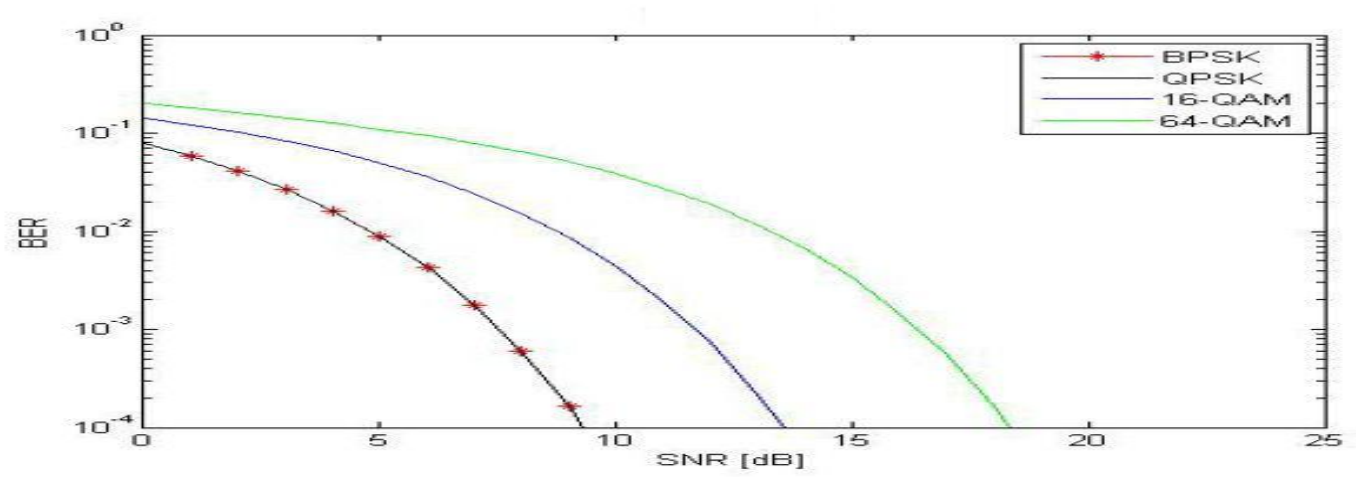

Fig 4: BER vs. SNR of SC-FDMA with BPSK, QPSK, 16QAM

The above figures (fig4\&fig5) shows signal-to-noise ratios of OFDMA and SC-OFDMA with different modulation schemes like BPSK, QPSK, 16-QAM, 64-QAM.

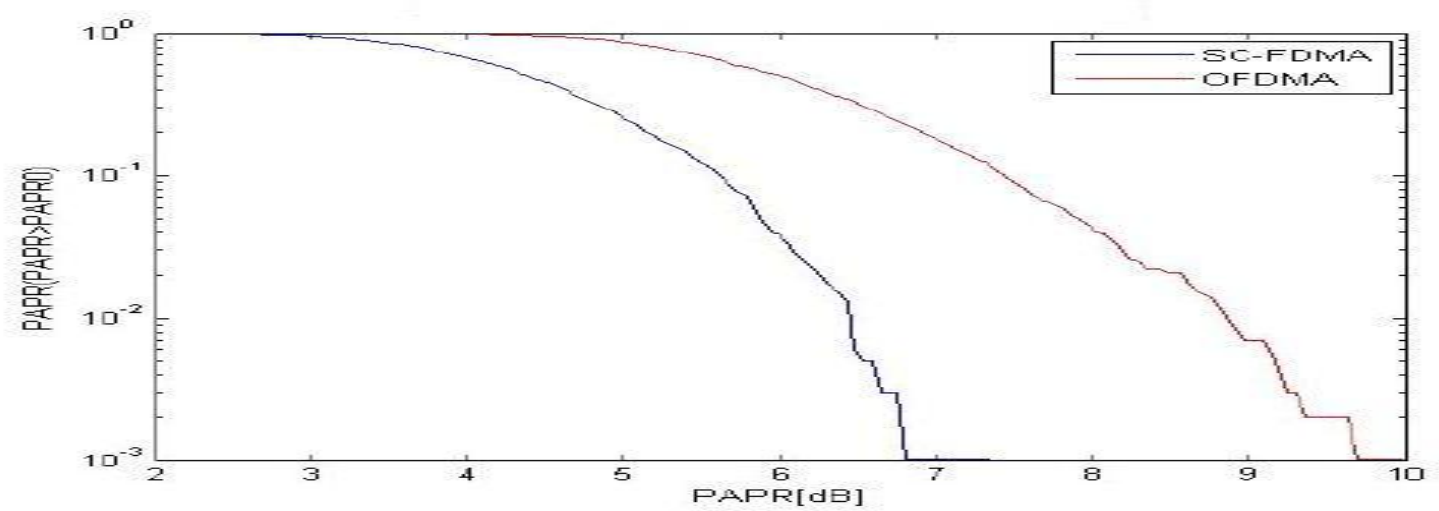

Fig 5: PAPR of OFDMA and SC-FDMA for BPSK.

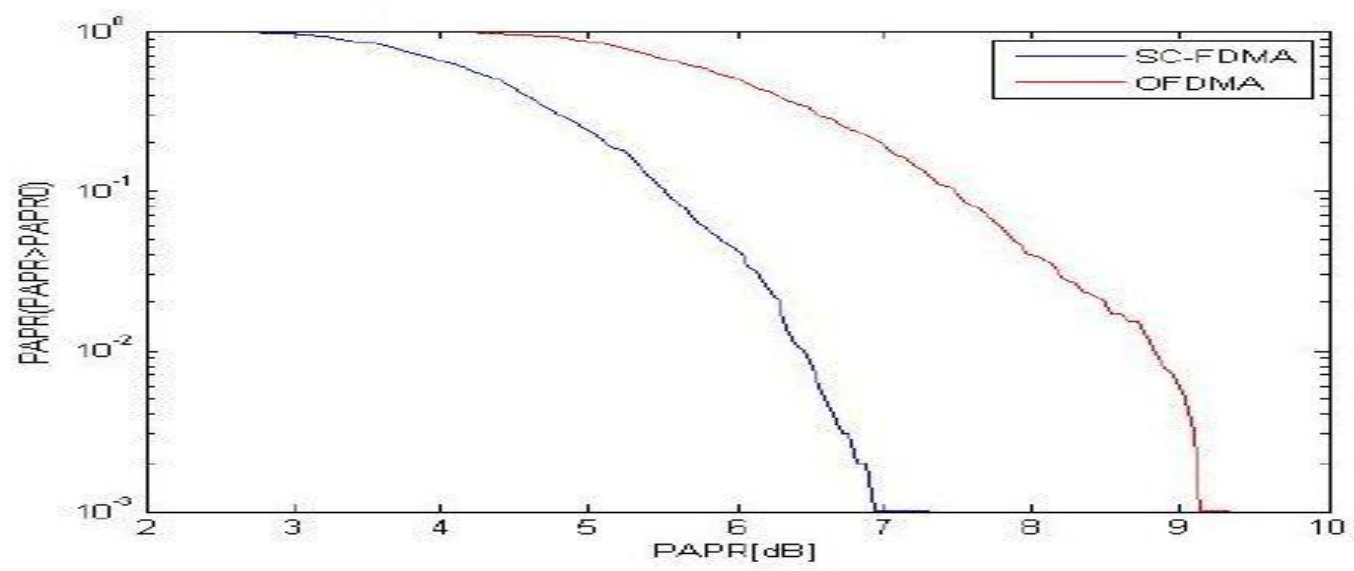

Fig 6: PAPR of OFDMA and SC-FDMA for QPSK.

The above figures (fig5\&fig6) explains peak- to- average power ratios of OFDMA and SC-FDMA with modulation schemes of BPSK and QPSK. 


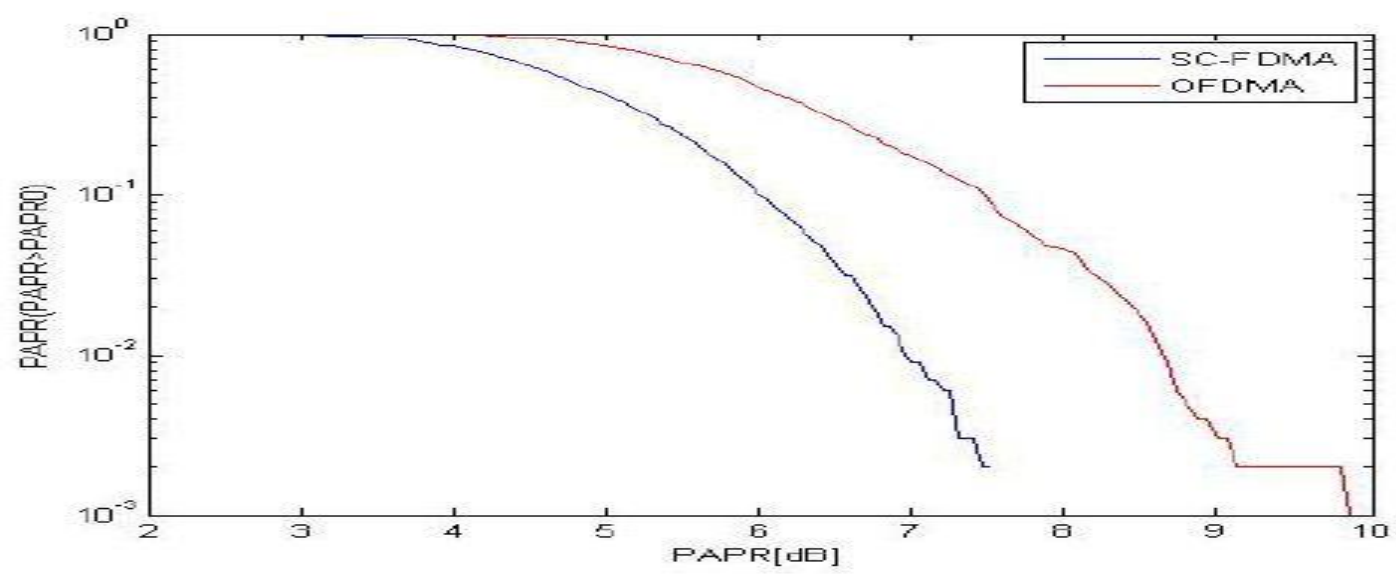

Fig 7: PAPR of OFDMA and SC-FDMA for 16-QAM.

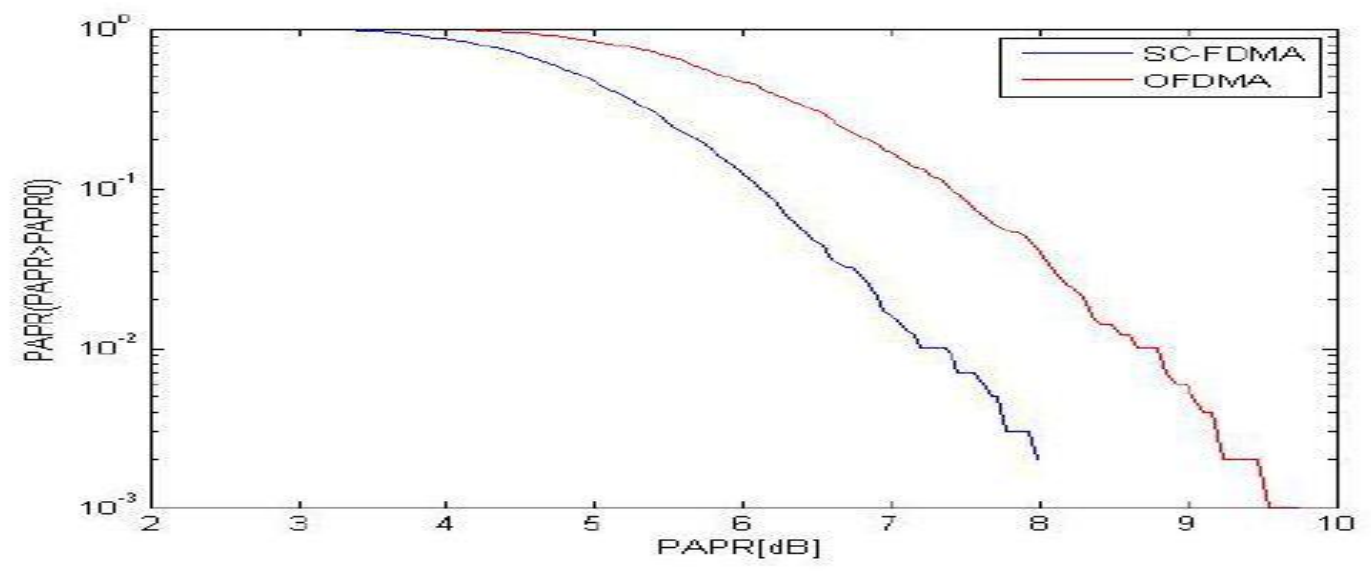

Fig 8: PAPR of OFDMA and SC-FDMA for 64-QAM.

The above figures (fig7\&fig8) shows PAPR of OFDMA and SC-FDMA with modulation schemes of 16 QAM and 64 QAM

\section{Conclusion}

For any data link to indicate the performance of the system BER is important parameter. The uplink and down link transmission modes used in LTE system increasing of the BER is observed for a fixed value of SNR for higher order modulation methods(16-QAM and 64-QAM) from the simulated results. In other way, at receiver BPSK and QPSK (i.e. lower order modulation schemes) experience less BER at receiver thus in terms of BER and SNR lower order modulations improves performance of the system[1]. By considering efficiency of bandwidth for these modulation methods within a given band width more data houses linked to lower order modulation techniques. Thus, there exists a tradeoff between BER and bandwidth efficiency among these modulation schemes used in LTE. Hence modulation techniques of different kind plays crucial role based on these results.

In LTE uplink transmission at the user end (i.e. Portable devices) power consumption is a vital issue. It can be decided that the methods of higher order modulations have an impact on the PAPR of both OFDMA and SC-FDMA. The PAPR increases in SC-FDMA and slightly decreases in OFDMA for higher order modulation schemes. The overall value of PAPR in SC-FDMA is still less than that of OFDMA in all modulation schemes, and that is why it has been adopted for uplink transmission in LTE system. Based on the results obtained, it can be concluded to adopt low order modulation scheme i.e. BPSK, QPSK and 16-QAM for uplink in order to have less PAPR at user end. In nutshell, SC-FDMA is more power efficient.

\section{References}

[1] 3GPP, "3rd Generation Partnership Project, Technical specification group radio access network", Physical channels and modulation (Release 8), 3GPP TS 36.211.

[2] N. Arshad, M. A. Jamal, Dur E. Tabish \& S. Saleem, "Effect of Wireless Channel Parameters on Performance of Turbo Codes", Advances in Electrical Engineering Systems (AEES), Vol. 1, No. 3, pp. 129-134, 2012. 
[3] 3GPP, "ITU Library and archive services", URL http://www.itu.int/en/history/overview/Pages/history.aspx cited on 12th September, 2013.

[4] J. Lee, J. K. Han and J. Zhang, "MIMO Technologies in 3GPP LTE and LTE Advanced", EURASIP Journal on Wireless Communications and Networking, 2009.

[5] Y. Yang, H. Hu, J. Xu \& G. Mao, "Relay Technologies for WiMAX and LTE-Advanced Mobile Systems", IEEE Communication Magazine, October, 2009.

[6] International Telecommunications Union, "IMT-Advanced Submission and Evaluation Process", URL http://www.itu.int/ITUR/index.asp?category=study-groups\&rlink=rsg5- imt-advanced \& lang=en cited on 12 th September, 2013.

[7] D. Astely, E. Dahlman, A. Furuskar, Y. Jading, M. Lindstrom \& S. Parkwvall, "LTE: the evolution of mobile broadband", IEEE Communication Magazine, April, 2009.

[8] 3GPP, "3rd Generation Partnership Project, Technical specification group radio access network", Physical channels and modulation (Release 8), 3GPP TS 36.201.

[9] T. Hong, "OFDM and its wireless applications: A survey", IEEE transactions on Vehicular Technology, Vol. 58, Issue 4, pp. 16731694, May 2009

[10] 3GPP, "3rd Generation Partnership Project, Technical specification group radio access network", Multiplexing and Channel Coding (Release 8), 3GPP TS 36.212.

[11] 4G: The What, Why and When- The worldwide adoption and growth of wireless are the fastest technological achievements in history- Tellabs. www.tellabs.com/solutions/ mobilebackhaul .

[12] Video-centric Network Coding Strategies for 4G Wireless Networks: An• Overview* by Marie-José Montpetit and Muriel Médard , MIT Research Laboratory for Electronics.

[13] The 4G Technology V/S other G Technologies by Shipra Gupta, Supriya Shakya, • Dept Of CSE, R.K.G.I.T.W,UP.

[14] Analysis of SIP-based mobility management in 4G wireless networks by Nilanjan• Banerjee*,Wei Wu, Kalyan Basu, Sajal K. Das, Center for Research in Wireless Mobility and Networking (CReWMaN), Department of Computer Science and Engineering, The University of Texas at Arlington, Arlington, TX 76019-0015, USA 